\title{
Low-power Slice Selective Imaging of Broad Signals
}

\author{
Weiqi Yang ${ }^{1}$, Jae-Seung Lee ${ }^{1,2}$, Boris Kharkov ${ }^{1}$, Andrew Ilott $^{1}$, Alexej \\ Jerschow $^{1}$
}

\section{Imaging of solid}

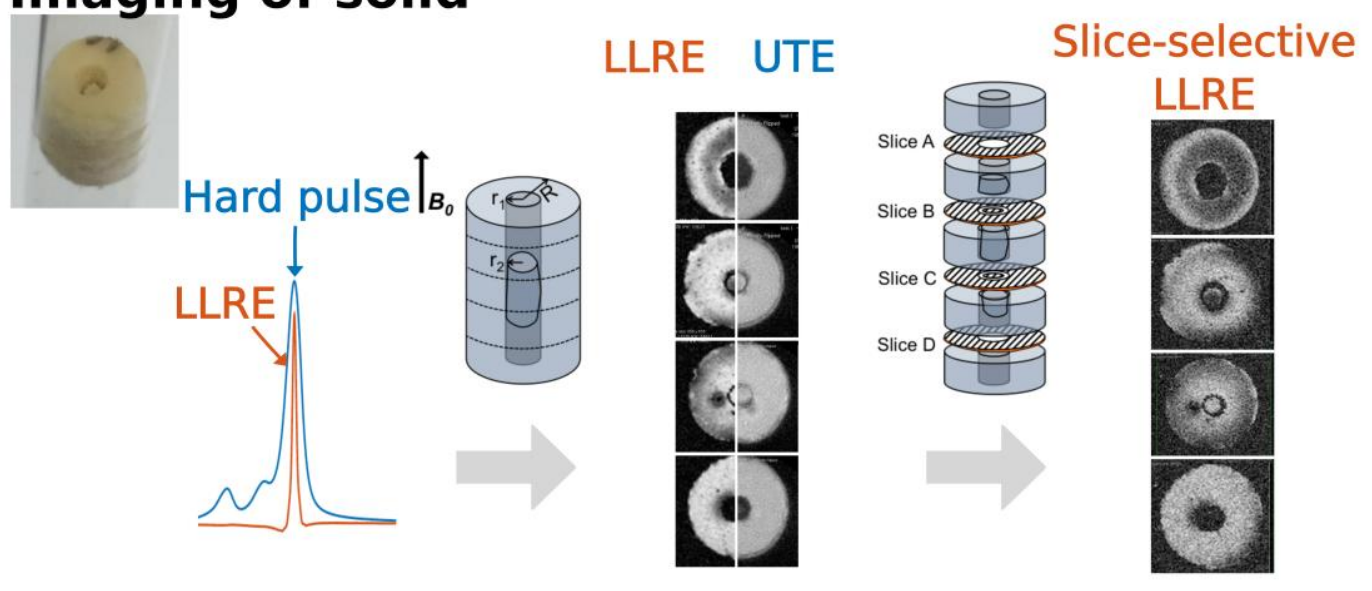

${ }^{1}$ Department of Chemistry, New York University, New York, NY, United States

${ }^{2}$ Department of Radiology, New York University Langone Medical Center, New

York, NY, United States

${ }^{*}$ Corresponding Author

Alexej Jerschow: alexej.jerschow@nyu.edu 


\section{Abstract}

One of the major challenges in using magnetic resonance imaging (MRI) to study immobile samples, such as solid materials or rigid tissues like bone or ligaments, is that the images appear dark due to these samples short-lived signals. Although it is well known that narrowband signals can be excited in inhomogeneously-broadened lines, it is less well known that similar effects can be observed in dipolar-broadened systems. These long-lived signals have not been used much, mainly because their description frequently does not match intuition. While 3D imaging with these signals has previously been reported, here we focus on the demonstration of faster, 2D slice-selective imaging. The faster imaging provides more flexibility for visualizing these rigid objects, an approach that is not compatible with ultra-short echo time (UTE) techniques. We also focus on the frequently-encountered regime where the maximum power achievable for If pulses is significantly weaker than the linewidth. This regime is typically encountered in clinical MRI scanning or large volume setups. When compared to UTE and conventional slice-selective spin echo, this technique provides better representations of the sample, and provides higher signal-to-noise ratios than spin-echo techniques in both the high and low power regimes for the eraser sample.

\section{Introduction}

Magnetic resonance imaging (MRI) has become a widely used technique providing exquisite images with excellent contrast detail of soft tissue and anatomic structures [1, 2], and is increasingly used in materials science [3-7]. One of the major challenges in imaging solid materials or rigid tissues (such as bone and ligament) is that their signals 
decay very quickly (within $\sim 1 \mu \mathrm{s}-1 \mathrm{~ms}$ ), so they are mostly invisible in conventional MRI images [8-12]. Several advanced methods involving the use of specialized hardware have been developed for visualizing relatively short-lived signal components, but are frequently incompatible with in vivo constraints [8-14]. In solids or rigid tissues, the signals are short-lived, and the spectrum is $3-5$ orders of magnitude broader than in liquids. Therefore, when the gradient strengths are limited, like in in vivo imaging, the linewidth becomes the limiting factor for resolution [15].

Two main processes contribute to the short signal lifetimes in rigid samples. First, the magnetic susceptibility of the tissues may vary in space, giving rise to considerable magnetic field inhomogeneity [16]. Second, the restricted molecular motion inside the tissues can leave dipolar couplings and chemical shift anisotropy in effect. These interactions can result in extremely strong signal dephasing and correspondingly broad resonance lines.

While it is well known that narrowband signals can be excited from inhomogeneously-broadened lines, it has also been demonstrated that long-lived signals can be excited from systems with homogeneously broadened dipolar spectra by using either a long and weak single if irradiation or such irradiation in combination with a subsequent strong $\pi$ pulse [17-20]. We have previously referred to the first method as the long-lived response (LLR) technique, and the second as the long-lived response echo (LLRE) technique. Our previous work indicated that dipolar coupling complexity may play an important role in producing the LLR and LLRE signals [21]. This effect was demonstrated by diluting the proton concentration in the sample, which showed a nonlinear effect on the LLR and LLRE signals. This observation was incompatible with a 
description based solely on inhomogeneous broadening. The line narrowing afforded by this LLRE signal excitation is of great utility and could be used to enhance image resolution. Although these techniques have been implemented in 3D MRI [20, 21], showing improved resolution and power behavior, the implementation of direct slice selection is not obvious for signals arising in dipolar coupled systems.

Here, we demonstrate that the LLRE signal can be made slice-selective by applying a gradient during the $\pi$ pulse, making the method compatible with fast $2 \mathrm{D} \mathrm{MRI}$ applications. The approach is tested on samples of rubber materials, and their $2 \mathrm{D}$ images acquired with the slice-selective LLRE signals are compared to those from with conventional spin echo and UTE methods. We also include a description of the free induction decay obtained from the long-lived signals as a function of flip angle.

\section{LLR and LLRE Formation Theory}

To understand the origin of the long-lived signal, the collective evolution of all frequency components of the density operator has to be considered. Starting from equilibrium, where the magnetization is aligned with the magnetic field, a weak excitation pulse with duration $t_{p}$ gives rise to the FID signal calculated by

$$
M(t)=\operatorname{Tr}\left\{S_{+} \rho(t)\right\}, \rho(t)=\exp (-i H t) \rho\left(t_{p}\right) \exp (i H t), t>t_{p} .
$$

$\rho(t)$ is the density operator, $S_{+}$is an observable in a quadrature detection experiment, and $\mathrm{H}$ represents the full spin Hamiltonian, that describes free evolution of the system and includes internal spin interactions such as dipolar couplings and chemical shifts for all interacting spins. 
Following the analysis proposed by Khitrin [18], we introduce the spectral components

$$
S_{\omega}: \quad \exp (-i H t) S_{\omega}(t) \exp (i H t)=\exp \left(-i \omega S_{z} t\right) S_{\omega}(t) \exp \left(i \omega S_{z} t\right) .
$$

A spectral component $S_{\omega}$ is a part of the density operator that oscillates with frequency $\omega$ and in general is a linear combination of all possible single-quantum coherences for the spin system. Here, $\omega$ can take values from the set of spectral frequencies of the full spin Hamiltonian, $H$. Let us calculate the spectral components for an inhomogeneously broadened line, e.g., the signal of water in the presence of magnetic field gradient. A homogeneously broadened line can be analyzed in the same way, except that the linear response regime would have to be used[18]. The rotating frame Hamiltonian during the weak excitation pulse is given by[18]

$$
H_{1}(\boldsymbol{r})=\omega_{1} S_{y}+\omega(\boldsymbol{r}) S_{z} .
$$

The term $\omega(\boldsymbol{r})$ is a function of the spatial coordinates and describes the nonuniformity of the local static magnetic field, $B_{0}$. The pulse applied to the initial $z$-magnetization leads to its rotation around the tilted effective field, $\omega_{\text {eff }}=\sqrt{\omega_{1}^{2}+\omega^{2}}$, whose direction is specified by a unit vector $\boldsymbol{u}=\left\{u_{x}, u_{y}, u_{z}\right\}$, leading to

$$
\exp \left(-i H_{1} t_{p}\right) S_{z} \exp \left(i H_{1} t_{p}\right)=u_{y} \sin \theta S_{x}+u_{y} u_{z}(1-\cos \theta) S_{y}+\left(\cos \theta+u_{z}^{2}(1-\cos \theta)\right) S_{z}
$$

where $\theta=\omega_{e f f} t_{p}$ is the effective flip angle. Taking into account that the $z$ component of magnetization does not contribute to the FID signal, we obtain the expression for the frequency components,

$$
S_{\omega}=\left[\frac{\sin \left(\omega_{1} t_{p} \sqrt{k^{2}+1}\right)}{\sqrt{k^{2}+1}}+i k \frac{1-\cos \left(\omega_{1} t_{p} \sqrt{k^{2}+1}\right)}{k^{2}+1}\right] S_{+},
$$


where $k(\boldsymbol{r})=\omega(\boldsymbol{r}) / \omega_{1}$. The conjugated term proportional to the operator $S_{-}$is ignored in Eq. (5). The FID can be calculated from the inverse Fourier transform of the $S_{\omega}$ distribution function [22],

$$
M(t)=\int_{-\infty}^{\infty} S_{\omega} e^{i \omega t} d \omega .
$$

while the spectrum is obtained from the Fourier transform of the FID signal, assuming that the acquisition starts at $t=0$,

$$
I(\omega)=\int_{0}^{\infty} M(t) e^{-i \omega t} d t .
$$

We note that the amplitudes of the individual spectral components $S_{\omega}$ are not equivalent to the spectral intensities $I(\omega)$ at a given frequency $\omega$, due to the different integration limits in Eq. (6) and (7).

The appearance of the FID calculated from Eq. (6) is shown in Fig. 1(a). Following Eq. (7), the spectrum for the LLR signal is calculated from the Fourier transform of the positive $(t>0)$ part of this signal, and is shown in Fig. 1(c). The effect of the hard $180^{\circ}$ pulse that follows the initial excitation pulse in the LLRE sequence is equivalent to changing the sign of the linear terms in the Hamiltonian (or time reversal in Eq. (6)) [7]. In this case, the calculated spectrum is the Fourier transform of the negative part of the FID in Fig. 1(a), yielding the characteristic lineshape in Fig. 1(b). These spectra match the published long-lived response lineshapes for inhomogeneously broadened water proton signals. [18] 



Fig.1 Numerical simulation of the long lived signals in water in the presence of a static field gradient corresponding to a variation in the Larmor frequency of $100 \mathrm{kHz}$ over the characteristic sample size. $\mathrm{B}_{1}=10 \mathrm{~Hz}$, and on-resonance flip angle $\omega_{1} t_{p}=\pi / 2$. The FID calculated as the reverse Fourier transform of Eq.(4) for LLR and LLRE experiments. A m pulse in LLRE sequence virtually reverses the direction of the arrow of time. (b) and (c): Spectra of LLRE and LLR signals, respectively.

For a system of many coupled spins, $S_{\omega}$ is proportional not only to the operator $S_{+}$but a combination of all single-quantum operators. In this case, the operator part of $S_{\omega}$ may be calculated from the following commutation equation 


$$
\left[H-\omega S_{z}, S_{\omega}\right]=0 .
$$

A quantitative description of the spectral components generation mechanism in a manyspin system with a homonuclear dipolar interaction network is not yet developed, mainly due to the difficulty in simulating systems with a sufficient number of coupled spins. A possible qualitative explanation, however, has been suggested and the similarity of the signals obtained in inhomogeneous and homogeneous systems have been demonstrated $[17,18]$. It has been suggested that a non-vanishing chemical shift difference between dipolar coupled spins is essential to the visibility of the effect.

As long as the spin Hamiltonian commutes with the operator $I_{z}$ and the condition in Eq. (8) is fulfilled, additional offset terms imposed by the applied gradients lead to the same frequency shift for all spectral components $S_{\omega}$ at a given spatial position. This observation means that despite the nature of the broadening, once a long-lived signal is excited by LLR, the static field gradients may be used in the same manner as is done in conventional MRI.

\section{Materials and Methods}

\section{Sample preparation}

From a piece of eraser (AXP96318, M\&G eraser, China), a large hollow cylinder (outer radius $R=4.30 \mathrm{~mm}$, inner radius $r_{1}=1.05 \mathrm{~mm}$, height $=10 \mathrm{~mm}$ ) and a smaller and shorter cylinder (radius $r_{2}=0.75 \mathrm{~mm}$ ) were prepared, and placed in a $10 \mathrm{~mm}$ NMR tube. Four different slices images were acquired using the spin echo method, LLRE3D and the slice selective LLRE methods. 
NMR and MRI Measurements

All the MRI and NMR experiments were performed on a Bruker Avance I spectrometer (Bruker, MA, USA) operating at $400.13 \mathrm{MHz}$ for ${ }^{1} \mathrm{H}$, equipped with a microimaging accessory. Images were collected with a Bruker Micro2.5 gradient assembly and imaging probe in combination with a $15 \mathrm{~mm}$ i.d. coil (Bruker Biospin $\mathrm{GmbH}$, Germany). The coil has a uniform excitation profile over a length of $15 \mathrm{~mm}$, with a total profile extending to $23 \mathrm{~mm}$. The samples were carefully aligned at the center of the coil to ensure full and uniform signal excitations across their full volumes. For conventional UTE and spin echo MRI experiments, the pulse sequences of Ultrashort TE(UTE) [23], UTE3D [24] and Multi-Slice Mullti-Echo (MSME) [25] provided in Paravision 5.1 (Bruker, Biospin, Germany) were used. For LLRE experiments, the imaging was implemented in Topspin 2.1 (Bruker, Germany).

\section{UTE method}

The 2D UTE sequence consisted of a slice-selective RF pulse followed by 2D radial ramp sampling. The size of the data matrix was $128 \times 128$, the pixel size $102 \times 102$ $\mu \mathrm{m}^{2}$, and the slice thickness $1 \mathrm{~mm}$. TE and TR were $0.424 \mathrm{~ms}$ and $5 \mathrm{~s}$, respectively. For the signal excitation, a $0.5950 \mathrm{~ms}$ long Gaussian pulse with a flip angle of $90^{\circ}$ was applied. We also performed a scan in which the maximum rf amplitude $\left(\gamma B_{1} / 2 \pi\right)$ of the Gaussian pulse was limited to $1000 \mathrm{~Hz}$ in order to test constraints that would be found on a clinical MRI system. The duration and flip angle of the Gaussian pulse were 0.6073 $\mathrm{ms}$ and $90^{\circ}$ in this case. The size of the data matrix, the pixel size, and the slice 
thickness were kept the same as above. TE and TR were $0.43 \mathrm{~ms}$ and $5 \mathrm{~s}$, respectively. The maximum gradient strength was $38.4 \mathrm{G} / \mathrm{cm}$ along the slice-selective direction and $95.9 \mathrm{G} / \mathrm{cm}$ along the $x$ and $y$ encoding directions. The number of projections was 402 .

The 3D UTE imaging was performed by a 3D radial FID sampling sequence using a square pulse of duration $0.02 \mathrm{~ms}$ with a flip angle of $5^{\circ}$. The size of the data matrix was $128 \times 128 \times 128$, the voxel size $102 \times 102 \times 234 \mu \mathrm{m}^{3}$, TE $0.02 \mathrm{~ms}$, and TR $8.0 \mathrm{~ms}$. Only one scan was taken per $k$-space readout in all experiments. The number of projections was 51360 .

Spin echo method

A slice-selective, 2D spin echo sequence was implemented using the multi-slice multiecho (MSME) method with 128 averages. The size of the data matrix was $128 \times 128$, the pixel size $102 \times 102 \mu \mathrm{m}^{2}$, and the slice thickness $1 \mathrm{~mm}$. TE and TR were $8.01 \mathrm{~ms}$ and $1 \mathrm{~s}$, respectively. We also performed a scan with the rf amplitude $\left(\gamma \mathrm{B}_{1} / 2 \pi\right)$ of the refocusing pulse limited to $1000 \mathrm{~Hz}$ to test power-limited conditions. Here, data matrix, the pixel size, and the slice thickness were kept the same as above, but TE and TR were $14 \mathrm{~ms}$ and $1 \mathrm{~s}$ respectively, and 128 averages were used. The RF pulse shape was a normalized sinc function on the $[-\pi-\pi]$ interval. The maximum gradient strength was $54.7 \mathrm{G} / \mathrm{cm}$ along the slice selection direction, $24.0 \mathrm{G} / \mathrm{cm}$ along the readout direction, and $13.4 \mathrm{G} / \mathrm{cm}$ along the phase-encoding direction.

\section{LLRE method}


The excitation of a LLRE signal was performed by a long and weak rf irradiation followed by a strong $\pi$ pulse, which was phase-shifted by $\pi / 2$ relative to the weak rf irradiation (Fig. 2(a)). The weak pulse was optimized to give the maximum signal. Its duration and rf amplitude $\left(\mathrm{\gamma B}_{1} / 2 \pi\right)$ were $6 \mathrm{~ms}$ and $27 \mathrm{~Hz}$, respectively. The rf amplitude $\left(\gamma B_{1} / 2 \pi\right)$ of the $\pi$ pulse was $14 \mathrm{KHz}$.

The 3D LLRE sequence consisted of the excitation of the LLRE signal followed by a 3D gradient-echo 3D acquisition. The $128 \times 128 \times 128$ data matrix covered a FOV of $13 \times 13 \times 16 \mathrm{~mm}^{3}$ with TR $=5 \mathrm{~s}$ and a slice thickness of $132 \mu \mathrm{m}$. The maximum strength of the applied gradients was $29.8 \mathrm{G} / \mathrm{cm}$ for the read-out gradient and 18.8 $\mathrm{G} / \mathrm{cm}$ for the phase-encoding gradient. The echo time of $3.90 \mathrm{~ms}$ for the LLRE method is defined as the time between the middle of the soft rf excitation pulse and the peak of the signal induced in the coil.

The slice-selective 2D LLRE imaging sequence consisted of the excitation of the LLRE signal, a slice selection gradient, and a 2D gradient echo readout (Fig. 3(b)). The slice selection is achieved using a slice selection gradient during the second pulse that is now a soft $\pi$ pulse to refocus the spins in the selected plane only. The same sincshaped pulse of $1 \mathrm{~ms}$ duration was used for the soft $\pi$ pulse. For the eraser sample (maximum excitation power of $\pi$ pulse $=2800 \mathrm{~Hz}$, Fig. 3 (c)), the $128 \times 128$ data matrix covers a FOV of $13 \times 13 \mathrm{~mm}^{3}$. A $0.283 \mathrm{~mm}$ slice was used. The strengths of the applied gradients were $38.7 \mathrm{G} / \mathrm{cm}$ for the read-out gradient, $28.4 \mathrm{G} / \mathrm{cm}$ for the phase-encoding gradient, and $29.8 \mathrm{G} / \mathrm{cm}$ for the slice selection gradient. The image was obtained with $\mathrm{TR}=5 \mathrm{~s}$ and $\mathrm{TE}=7 \mathrm{~ms}$. For a quantitative evaluation of the LLRE method, the $128 \times$ 128 data matrix covers a FOV of $13 \times 13 \mathrm{~mm}^{3}$. A $1 \mathrm{~mm}$ slice was used. The strength of 
applied gradients is $35.7 \mathrm{G} / \mathrm{cm}$ for the read-out gradient, $28.9 \mathrm{G} / \mathrm{cm}$ for the phaseencoding gradient and $3.6 \mathrm{G} / \mathrm{cm}$ for the slice selection gradient. The image was obtained with a TR of $1 \mathrm{~s}$ and TE of $5.0 \mathrm{~ms}$. We also performed a scan in which the maximum rf amplitude $\left(\gamma B_{1} / 2 \pi\right)$ of the $\pi$ pulse was limited to $1000 \mathrm{~Hz}$, the image was obtained with a TR of $1 \mathrm{~s}$ and TE of $6.8 \mathrm{~ms}$.

\section{Results and Discussion}

The Fourier-transformed spectrum of a LLRE signal from the eraser sample is shown in Fig. 2(b), together with the conventional ${ }^{1} \mathrm{H}$ NMR spectrum acquired with a $90^{\circ}$ hard pulse. With the LLRE excitation, a single peak generally appears on the LLRE spectrum at the same frequency offset as the weak rf irradiation, and its linewidth is significantly narrower than that of the conventional ${ }^{1} \mathrm{H}$ spectrum. For example, the linewidths of the spectra shown in Fig. 2(b) are $176 \mathrm{~Hz}$ and $856 \mathrm{~Hz}$, respectively for the LLRE and conventional spectra. The signal intensity ratio between the LLRE and conventional spectra is 1:2.3. Although LLRE signals are weaker than the signals excited by a hard pulse, the decreased signal intensity can be compensated for by their slower decay in time.
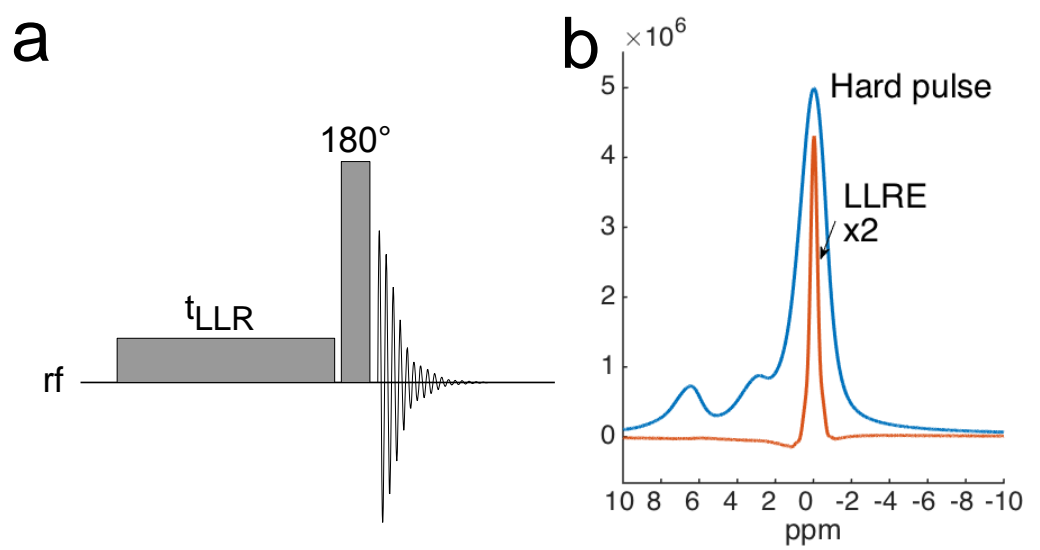
Fig. 2 (a) The LLRE pulse sequence consisting of a weak pulse and a $180^{\circ}$ pulse. For the weak pulse, $\mathrm{t}_{\mathrm{LLR}}=6 \mathrm{~ms}$ and $\gamma \mathrm{B}_{1} / 2 \pi=27 \mathrm{~Hz}$. For the $180^{\circ}$ pulse, a $36.7 \mu \mathrm{s}$-long hard pulse with $\gamma B_{1} / 2 \pi=13624 \mathrm{~Hz}$ was used for the LLRE spectrum shown in (b). (b) Conventional and LLRE ${ }^{1} \mathrm{H}$ NMR spectra of an eraser sample. The conventional spectrum was obtained with a $90^{\circ}$ hard pulse $\left(\gamma B_{1} / 2 \pi=13624 \mathrm{~Hz}\right)$. The LLRE spectrum was acquired with the pulse sequence shown in (a).

The slice selection is achieved using a gradient during the $\pi$ pulse, which thus acts on spins in the selected plane only (Fig. 3(b)). Four different slices were chosen to verify the slice selection and compare to the 3D LLRE and UTE imaging, as illustrated in Fig. 3(a). Slices $A$ and $D$ were chosen at $4.22 \mathrm{~mm}$ and $-3.28 \mathrm{~mm}$ away from the isocenter, where only the outer hollow outer cylinder is present. Slices B and C were set at $2.34 \mathrm{~mm}$ from the isocenter and at the isocenter itself, respectively, hence both the inner and outer cylinders should show up. The images of the chosen slices acquired with 2D slice-selective LLRE, 3D LLRE, and 3D UTE sequences are shown in Fig. 3(b). The LLRE3D images appear superior to the UTE images of the same nominal resolution, showing more structural details and a clearer gap between the inner and outer material. The slice-selective LLRE images have lower signal-to-noise ratio than the other two, as expected considering that only spins on the selected plane contribute to the signal. This result proves that the slice selection is implemented successfully in the LLRE pulse sequence. Direct slice-selective excitation does not work with this and similar samples, because the gradient localization is convoluted with the inherent 
broadening. By contrast, a slice-selective $\pi$ pulse preserves the long-lived signal, while also providing the slice localization.

a
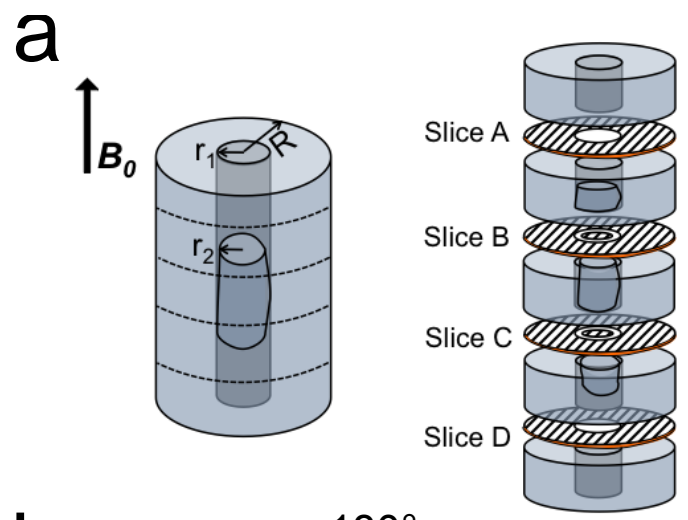

C

b
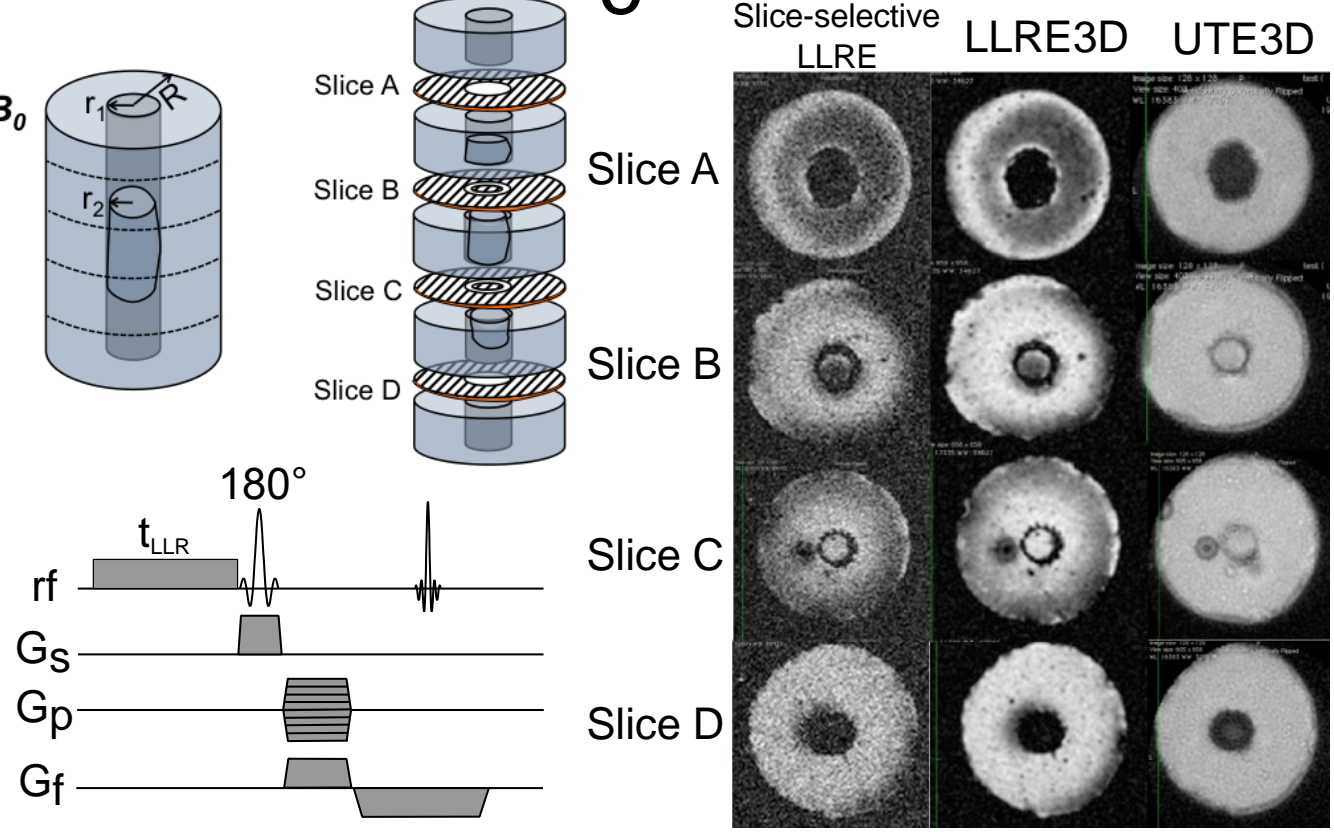

Fig. 3 Slice-selective LLRE imaging of an eraser sample. (a) Schematic and slices of the eraser sample used in the MRI experiments. $\left(R=4.3 \mathrm{~mm}, r_{1}=2.1 \mathrm{~mm}, r_{2}=1.5 \mathrm{~mm}\right)$ (b) Slice-selective imaging sequence using LLRE as the signal excitation method. For the first pulse, $t_{L L R}=6 \mathrm{~ms}$ and $\gamma B_{1} / 2 \pi=27 \mathrm{~Hz}$. The $180^{\circ}$ pulse was implemented by a 1 ms sinc pulse with $\gamma B_{1,(\max )} / 2 \pi=2816 \mathrm{~Hz}$. (c) Images obtained with slice-selective LLRE imaging, along with slices extracted from 3D LLRE and UTE scans.

For a quantitative evaluation of the LLRE method, the images for slice $\mathrm{C}$ were compared with a slice-selective spin echo sequence, and a 2D UTE sequence as well, 
under the same nominal resolution and repetition time. As illustrated in Fig. 4, the LLRE image manifests the finer details and higher sensitivity than the other two images. The quality of the image was assessed via the signal-to-noise ratio (SNR) determined by

$$
S N R=\frac{S_{R O I}}{S D_{\text {air }}}
$$

where $S_{\mathrm{RO}}$ is the mean signal in the large circular region covering most of the test object and $\mathrm{SD}_{\text {air }}$ is the average of the standard deviations in two smaller regions placed over air [26]. The UTE image has the highest SNR (Table 1), however, it is blurry in details compared to the other two. This effect is also seen from the cut view of the images along the horizontal line located in the center of images (bottom of Figure 4). The LLRE image clearly shows the gap between the inner and the outer material, the signal of which is almost as low as the noise level outside, while the spin echo and UTE images show a blurry gap. This is expected, because during the readout, the gradient localization is convolved by the spectral width of the excited signal. While one could remedy this situation by applying a stronger gradient, such strong gradients are not always available on MRI scanners, and sensitivity would be degraded. The sliceselective LLRE image has a higher SNR than the spin echo image and represents the object's features more clearly than the UTE image, due to its narrower spectral width. 


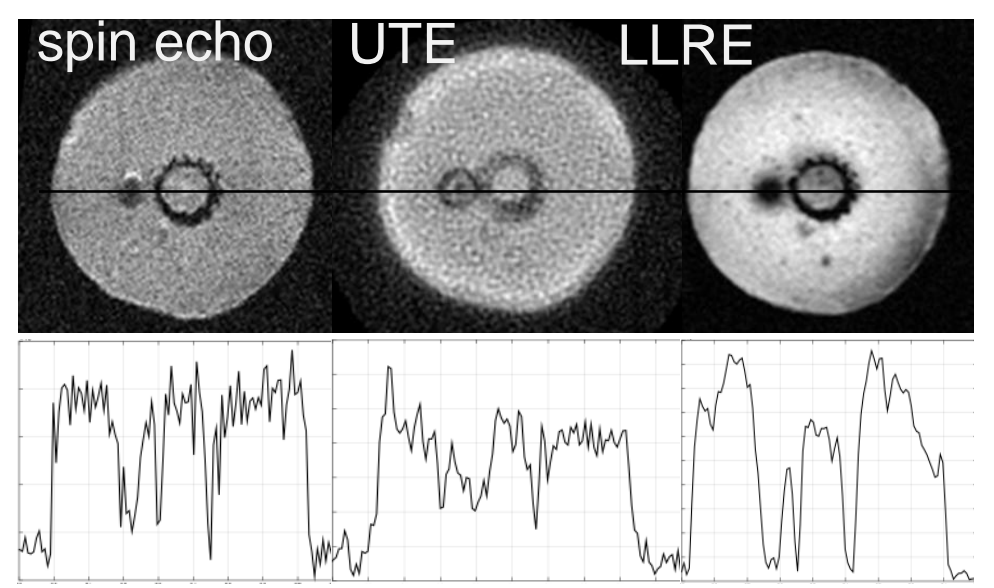

Fig.4 Top: 2D slices from an eraser sample acquired with the spin echo, UTE2D, and slice-selective LLRE imaging sequences. The same nominal resolution, data matrix, FOV, and repetitions time were used. The maximum RF amplitude for each method is around $2800 \mathrm{~Hz}$. (For both spin-echo sequence and LLRE sequence, the amplitude of second pulse (the $\pi$ pulse) is set as the maximum.) Bottom: A cut view of images for each method along the horizontal line.

Table 1. Signal-to-noise ratio calculation for MRI methods

\begin{tabular}{lccc}
\hline & Spin echo & UTE & LLRE \\
\hline $\mathrm{S} / \mathrm{N}$ ratio (maximum RF amplitude $=2800 \mathrm{~Hz}$ ) & 1.13 & 13.46 & 4.46 \\
$\mathrm{~S} / \mathrm{N}$ ratio (maximum RF amplitude $=1000 \mathrm{~Hz}$ ) & 0.57 & 8.97 & 1.7 \\
\hline
\end{tabular}

On clinical scanners, the power of if pulses power is significantly restricted for safety reasons. On instruments designed for materials imaging, if pulse power may also be restricted, especially when large volume coils are used. For the purposes of testing 
this situation, we repeated the experiments with the maximum rf amplitude $\left(\gamma_{1} / 2 \pi\right)$ limited to $1000 \mathrm{~Hz}$. The images are shown in Fig. 5. Compared to the images acquired with the 2D UTE and spin echo with slice selection, the slice-selective LLRE method clearly results in higher resolution and higher sensitivity than the spin-echo method (Table 1). The cut view in Fig. 5 also indicates better signal to noise ratio and detail representation of LLRE signal when compared with the other two methods.

The same sequence as well as the spin echo and UTE3D sequences were applied to a hollow eraser sample containing water, the result of which is shown in Fig. 6. As expected, the spin echo image exhibited only the water compartment. The UTE image showed both the solid compartment as well as the water compartment. By contrast, the LLRE image displayed the solid compartment exclusively with the water compartment remaining invisible. This technique, therefore, shows potential for sliceselective imaging of objects with broad resonance lines in both the high- and low-power limits. Certain distortions and modifications of image contrast can be expected due to $B_{0}$ inhomogeneities, although the frequency of the LLRE signal is determined by the frequency of the weak pulse, not by the local Larmor frequency [27]. Such effects can be mitigated by using an excitation pulse well within the center of the broad resonance, especially in the presence of a line with broad linewidth. In the samples considered here, the intrinsic lineshape was much larger than typical inhomogeneities expected from susceptibility differences.

When power and gradient strength are unlimited, UTE, ZTE, and single-point imaging (SPI) currently remain the methods of choice, however. 

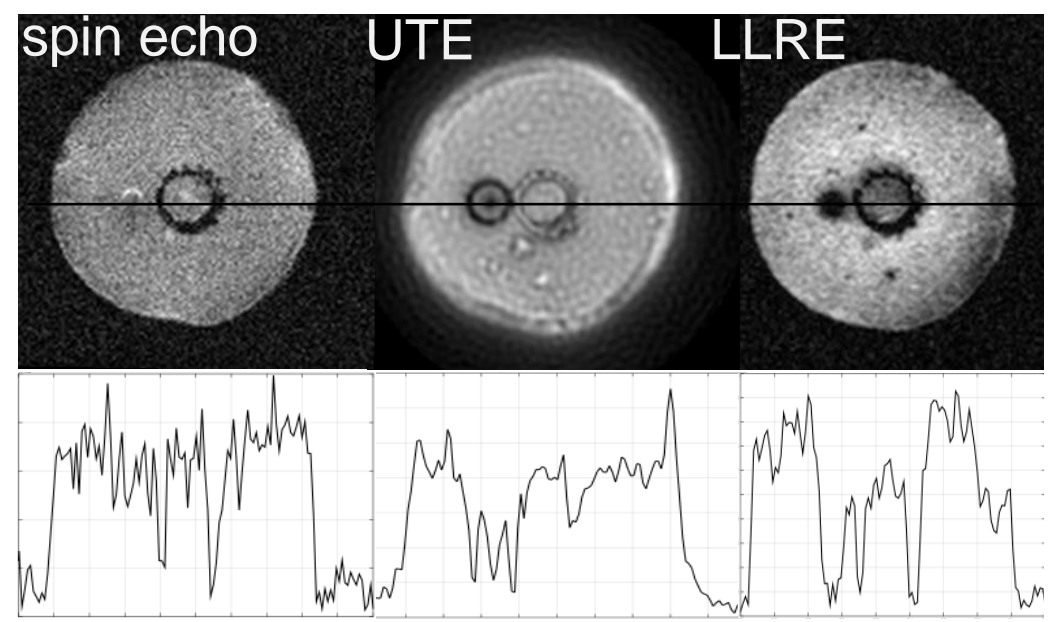

Fig.5 Top: 2D slices from an eraser sample acquired with the spin echo, UTE2D, and slice-selective LLRE imaging sequences. The same nominal resolution, data matrix, FOV, and repetitions time were used. The maximum RF amplitude for each method is $1000 \mathrm{~Hz}$. (For both spin-echo sequence and LLRE sequence, the second pulse ( $\pi$ pulse) is set as the maximum.) Bottom: A cut view of images for each method along the horizontal line.

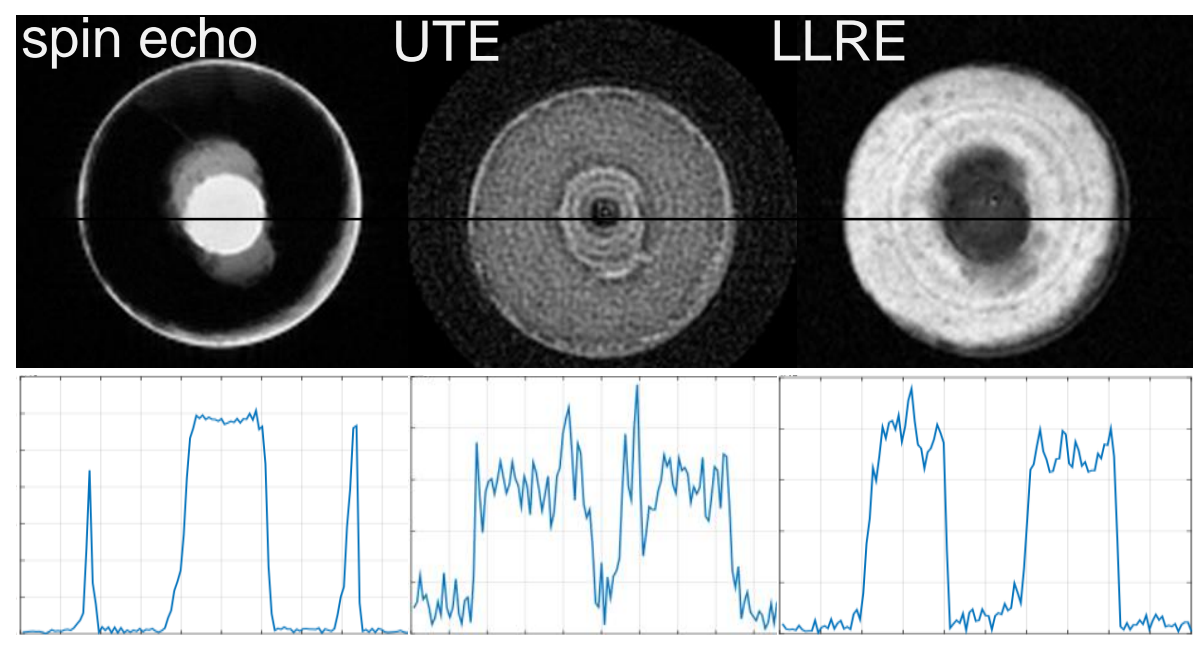

Fig. 6. Top: 2D slices from a hollow eraser cylinder containing un-doped water acquired with the slice-selective spin echo, UTE3D and slice-selective LLRE imaging sequence. Bottom: A cut view through the images for each method along the horizontal line. 


\section{Conclusions}

We have examined here the possibility of low-power excitations for slice-selective imaging in samples with strong line broadening due to either inhomogeneous or dipolar broadening mechanisms. We demonstrate that long-lived signals in rigid samples can be excited, which can subsequently be inverted by slice-selective if pulses. This procedure is implemented for an imaging method where we compare its performance with the 3D UTE, 2D UTE, and slice-selective spin-echo sequences. In addition, we examine here the power-limited case, which is important for MRI scanners or large volume and materials applications. It is seen that the slice-selective LLRE method clearly demonstrates better images with higher sensitivity under both the high and low power regimes. These results show that the slice-selective LLRE method may have potential for imaging semi-solids or biological rigid tissues containing water, especially under power-limited conditions. The method may not be applicable to all solid-like materials, however, for certain conditions should be met to excite the LLRE signal. At present the understanding about the origin and mechanism of LLR excitation is incomplete, and further efforts in this area could help clarifying the nature of the obtained image contrast. The 2D method itself could be further improved by the use of a multiple-slice acquisition scheme.

\section{Acknowledgements}


We acknowledge funding from the U.S. National Science Foundation, award No. CHE 1412064 for methodology development, and NIH R01EB016045 and K25AR060269 for imaging work.

\section{References}

[1] C. Westbrook, C.K. Roth, J. Talbot, MRI in practice. Hoboken, in, NJ: John Wiley \& Sons, 2011.

[2] M.A. Brown, R.C. Semelka, MRI: basic principles and applications, John Wiley \& Sons, 2011.

[3] D.E. Demco, B. Blümich, NMR imaging of materials, Curr. Opin. Solid State Mater. Sci., 5 (2001) 195-202.

[4] A.J. Ilott, S. Chandrashekar, A. Klöckner, H.J. Chang, N.M. Trease, C.P. Grey, L. Greengard, A. Jerschow, Visualizing skin effects in conductors with MRI: $7 \mathrm{Li} \mathrm{MRI}$ experiments and calculations, J. Magn. Reson., 245 (2014) 143-149.

[5] A.J. Ilott, N.M. Trease, C.P. Grey, A. Jerschow, Multinuclear in situ magnetic resonance imaging of electrochemical double-layer capacitors, Nat. Commun., 5 (2014). [6] M. Terekhov, D. Höpfel, MRI with the dipolar interaction refocusing techniques: analysis of the effectiveness for the solid-state polymers, Magn. Reson. Imaging, 22 (2004) 573-582.

[7] P.T. Callaghan, Principles of Nuclear Magnetic Resonance Microscopy, Clarendon Press, 1993.

[8] H.S. Rad, S.C.B. Lam, J.F. Magland, H. Ong, C. Li, H.K. Song, J. Love, F.W. Wehrli, Quantifying cortical bone water in vivo by three- dimensional ultra- short echo- time MRI, NMR Biomed., 24 (2011) 855-864.

[9] Y. Wu, M.I. Hrovat, J.L. Ackerman, T.G. Reese, H. Cao, K. Ecklund, M.J. Glimcher, Bone matrix imaged in vivo by water- and fat- suppressed proton projection MRI (WASPI) of animal and human subjects, J. Magn. Reson. Imaging, 31 (2010) 954-963.

[10] G.H. Filho, J. Du, B.C. Pak, S. Statum, R. Znamorowski, P. Haghighi, G. Bydder, C.B. Chung, Quantitative characterization of the Achilles tendon in cadaveric specimens: $\mathrm{T} 1$ and $\mathrm{T}^{*}$ measurements using ultrashort-TE MRI at $3 \mathrm{~T}, \mathrm{Am}$. J. Roentgenol., 192 (2009) W117-W124.

[11] A. Techawiboonwong, H.K. Song, F.W. Wehrli, In vivo MRI of submillisecond T2 species with two-dimensional and three- dimensional radial sequences and applications to the measurement of cortical bone water, NMR Biomed., 21 (2008) 59-70. [12] W.C. Bae, J. Du, G.M. Bydder, C.B. Chung, Conventional and Ultrashort MRI of Articular Cartilage, Meniscus and Intervertebral Disc, Top. Magn. Reson. Imaging, 21 (2010) 275.

[13] K. Schieban, M. Weiger, F. Hennel, A. Boss, K.P. Pruessmann, ZTE imaging with enhanced flip angle using modulated excitation, Magn. Reson. Med., 74 (2015) 684693. 
[14] M. Weiger, D.O. Brunner, B.E. Dietrich, C.F. Muller, K.P. Pruessmann, ZTE imaging in humans, Magn. Reson. Med., 70 (2013) 328-332.

[15] M.J. Kim, A.K. Khitrin, Magnetic resonance imaging of objects with dipolarbroadened spectra using soft excitation pulses, Magn. Reson. Imaging, 23 (2005) 865869.

[16] J.A. Hopkins, F.W. Wehrli, Magnetic susceptibility measurement of insoluble solids by NMR: Magnetic susceptibility of bone, Magn. Reson. Med., 37 (1997) 494-500.

[17] A.K. Khitrin, Long-lived NMR echoes in solids, J. Magn. Reson., 213 (2011) 22-25.

[18] A.K. Khitrin, Selective excitation of homogeneous spectral lines, J. Chem. Phys., 134 (2011) 154502.

[19] A.K. Khitrin, V.L. Ermakov, B.M. Fung, NMR molecular photography, arXiv preprint quant-ph/0208136, (2002).

[20] A.K. Khitrin, V.L. Ermakov, B.M. Fung, Information storage using a cluster of dipolar-coupled spins, Chem. Phys. Lett., 360 (2002) 161-166.

[21] B. Zhang, J.-S. Lee, A. Khitrin, A. Jerschow, Long lived NMR signal in bone, J. Magn. Reson., 231 (2013) 1-4.

[22] R.R. Ernst, G. Bodenhausen, A. Wokaun, Principles of Nuclear Magnetic Resonance in One and Two Dimensions, Clarendon Press, Oxford 1987. 610 Seiten, 1988.

[23] J.A. Du, M. Carl, M. Bydder, A. Takahashi, C.B. Chung, G.M. Bydder, Qualitative and quantitative ultrashort echo time (UTE) imaging of cortical bone, J. Magn. Reson., 207 (2010) 304-311.

[24] W.C. Bae, P.C. Chen, C.B. Chung, K. Masuda, D. D'Lima, J. Du, Quantitative ultrashort echo time (UTE) MRI of human cortical bone: correlation with porosity and biomechanical properties, J. Bone Miner. Res., 27 (2012) 848-857.

[25] A. Mackay, K. Whittall, J. Adler, D. Li, D. Paty, D. Graeb, In-Vivo Visualization of Myelin Water in Brain by Magnetic-Resonance, Magn. Reson. Med., 31 (1994) 673-677. [26] M.J. Firbank, A. Coulthard, R.M. Harrison, E.D. Williams, A comparison of two methods for measuring the signal to noise ratio on MR images, Phys. Med. Biol., 44 (1999) N261.

[27] V. Antochshuk, M.-J. Kim, A. K. Khitrin. High-resolution NMR imaging of objects with dipolar-broadened spectra. J. Magn. Reson. 167 (2004) 133-137. 